\title{
Massive vagal schwannoma in an 11-year-old girl
}

\author{
Noah Feit ${ }^{1}$, Conall Fitzgerald ${ }^{2}$, Timothy McLean ${ }^{2}$, and Richard Wong ${ }^{2}$ \\ ${ }^{1}$ Weill Cornell Medicine \\ ${ }^{2}$ Memorial Sloan-Kettering Cancer Center
}

June 23, 2021

\begin{abstract}
We describe an unusual case of a young girl presenting with a large vagal schwannoma necessitating a transcervical-mandibulotomy approach for total tumor resection. The presentation is unique due to the size of the lesion, the patient's age, the operative approach, and molecular pathology.
\end{abstract}

\section{Introduction}

Vagal schwannomas are rare peripheral nerve sheath tumors arising from the vagus nerve. ${ }^{1}$ Typically diagnosed in adulthood at a median age of 44 years, ${ }^{2}$ pediatric vagal schwannomas are exceedingly uncommon. Tumors may arise intracranially or extracranially, with the parapharyngeal space being the most common extracranial site in the head and neck. ${ }^{3}$

Surgical resection remains the treatment of choice for vagal schwannomas. Described operative techniques include gross total resection or intracapsular enucleation. However, the merits of either approach are dependent on the lesion size, location and baseline nerve function.

We describe herein an unusual case of a young girl presenting with a large vagal schwannoma with an associated hypoglossal nerve palsy necessitating a combined transcervical-mandibulotomy approach for total tumor resection. The presentation is unique due to the size of the lesion, the patient's age, the operative approach required, as well as the paralysis of a cranial nerve that was not its nerve of origin.

\section{Case Report}

An 11-year-old female presented with an asymptomatic, right neck mass first noted 7 months prior. Physical examination was notable for a large right parapharyngeal space mass on palpation of the oropharynx and neck, with medialization of the right oropharynx. Atrophy and fasciculations of the right side of the tongue were seen, consistent with a right hypoglossal nerve paralysis. Flexible fiberoptic laryngoscopy demonstrated effacement of the right nasopharynx and oropharynx, with preserved vocal fold function. All other cranial nerves were intact.

Magnetic resonance imaging (MRI) of the neck highlighted a large ovoid mass in the right parapharyngeal space measuring $4.9 \mathrm{~cm} \times 3.7 \mathrm{~cm} \times 7.4 \mathrm{~cm}$. The tumor extended from the jugular foramen superiorly to the level of C5 inferiorly, but without intracranial extension. The mass caused acute displacement of the internal and external carotid arteries anteriorly, and compression of the internal jugular vein postero-laterally. T2-weighted MRI sequences demonstrated heterogeneous hyperintensity (Figures 1A and 1B). T1-weighted MRI sequences showed a hypointense lesion with homogeneous avid enhancement after administration of gadolinium-based contrast, consistent with schwannoma (Figure 1C). ${ }^{1}$ Genetic testing and Pediatric Brain and Nervous System Tumor Panel (Invitae; San Francisco, California) were negative for any germline mutations. Following multi-disciplinary review and extensive patient and family discussion, the decision was 
made to proceed with complete surgical excision, involving a combined transcervical-mandibulotomy approach (Figures 2A-2E).

Histopathologic examination of the tumor confirmed schwannoma with immunohistochemistry showing diffuse S100 and focal SMA positivity. Memorial Sloan Kettering Integrated Mutation Profiling of Actionable Cancer Targets (MSK-IMPACT), a next-generation sequencing assay of all protein-coding exons of 468 cancer genes, demonstrated TET1 deletion of exons 1 and 2.

The patient underwent an uncomplicated post-operative recovery. A temporary gastrostomy tube was sited to assist with nutritional supplementation during swallow rehabilitation. A right vocal fold injection augmentation with Prolaryn Plus (Merz Pharma GmbH \& Co. KGaA; Frankfurt, Germany) was performed with a good voice result. At 3 months post-operation, the patient had no significant dysphagia or aspiration and demonstrated a normal voice.

\section{Discussion}

Neurogenous tumors are rare amongst children, comprising only $2 \%$ of benign non-lymphadenomatous lesions. ${ }^{4}$ Pediatric cervical vagal schwannomas are especially rare. The median age of diagnosis is 44 years in vagal schwannoma patients undergoing surgery. ${ }^{2}$ The median maximum diameter of these tumors is 5 centimeters. ${ }^{2}$ Cervical schwannomas grow at a rate of 3 millimeters per year, and usually only become symptomatic once large enough to cause compressive symptoms. ${ }^{1}$ Our patient's young age of 11 years old, in tandem with the large size of the lesion, makes her presentation extraordinarily uncommon.

Schwannomas arise from a single nerve fascicle and may cause cranial nerve dysfunction by exerting pressure on other fibers of the affected or local cranial nerves. Reported series to date differ widely on the prevalence of cranial nerve deficits at presentation. Interestingly, our patient did not exhibit signs of vagus nerve dysfunction, despite the nerve being involved intraoperatively, while she did have a long-standing hypoglossal nerve palsy, seemingly due to extrinsic compression from the vagal schwannoma. Although schwannomas do not typically present with cranial nerve palsies, when they do, dysfunction is usually of the index cranial nerve. Cranial nerve palsy may be suggestive of compression in a confined space or malignant schwannoma. Our patient's presentation with a hypoglossal nerve palsy with intact vagal nerve function in the context of a benign vagal schwannoma is, therefore, unusual.

Surgical resection is the preferred treatment modality for vagal schwannoma, either with gross total resection or intracapsular enucleation. Gross total resection may be superior to enucleation with respect to cure, but often results in loss of ipsilateral vagal function distal to the lesion. Enucleation may have superior outcomes vis-à-vis preservation of vagal nerve function, but is generally reserved only for small schwannomas and is thought to be associated with higher rates of recurrence.

The transcervical approach is the most commonly employed technique to access vagal schwannomas. ${ }^{2}$ Our combined transcervical-mandibulotomy approach was necessitated by an inability to safely gain access to the superior aspect of the tumor due to close adherence to the jugular foramen/skull base and adjacent, displaced critical vascular structures. Mandibulotomy allowed for improved access, safe mobilization of the tumor, and dissection of vessels at the level of the skull base.

Vagal schwannoma can arise in the context of genetic syndromes, including Neurofibromatosis Type 2, Carney's Complex, and schwannomatosis, with mutations in NF2 , SMARCB1 ,LZTR1, PRKAR1a previously described. Genomic changes in these genes all lead to downstream alterations in the production or function of the protein merlin, which plays a role in a range of growth factor pathways. ${ }^{1}$ Our patient demonstrated no germline mutation or syndrome. Memorial Sloan Kettering Integrated Mutation Profiling of Actionable Cancer Targets (MSK-IMPACT) identified an isolated TET1 deletion of exons 1 and 2. TET1 plays a role in epigenetic tumorigenesis and may be implicated in schwannomatosis. ${ }^{5}$ The clinicopathologic significance of the alteration in the setting of pediatric vagal schwannoma is unclear.

\section{Conclusion}


We present a case of cervical vagal schwannoma that is novel in terms of the young age of the patient, the size of the lesion, the combined transcervical-mandibulotomy approach and the molecular pathology.

\section{References}

1. Sandler ML, Sims JR, Sinclair C, et al. Vagal schwannomas of the head and neck: A comprehensive review and a novel approach to preserving vocal cord innervation and function. Head Neck . 2019;41(7):2450-2466. doi:10.1002/hed.25758

2. Cavallaro G, Pattaro G, Iorio O, Avallone M, Silecchia G. A literature review on surgery for cervical vagal schwannomas. World J Surg Oncol . 2015;13:130. doi:10.1186/s12957-015-0541-6

3. Kamath PM, Dosemane D, Sreedharan SS, Majeed NA, Shenoy VS. Vagal schwannoma: A rare parapharyngeal tumour. J Clin Diagn Res . 2016;10(4):MD03-4. doi:10.7860/JCDR/2016/16391.7588

4. Connolly AA, MacKenzie K. Paediatric neck masses-a diagnostic dilemma. J Laryngol Otol . 1997;111(6):541-545. doi:10.1017/s0022215100137867

5. Mansouri S, Suppiah S, Mamatjan Y, et al. Epigenomic, genomic, and transcriptomic landscape of schwannomatosis. Acta Neuropathol . 141(1):101-116. doi:10.1007/s00401-020-02230-x

\section{Figure Legends}

\section{Figure 1}

Figures 1A and 1B: T2-weighted MRI demonstrated a large ovoid mass in the right parapharyngeal space splaying the carotid artery and its branches and effacing the internal jugular vein. The tumor was heterogeneously hyperintense relative to skeletal muscle.

Figure 1C: The lesion exhibits homogeneous avid enhancement after administration of gadolinium-based contrast, consistent with a radiologic diagnosis of schwannoma.

Figure 1D: Imaging 6 months post-operatively demonstrates no evidence of disease.

Figure 2

Figure 2A: The hypoglossal nerve (yellow vessel loop) is seen stretched and tented over the tumor, explaining the patient's preoperative hypoglossal nerve palsy. It was not possible to further mobilize the tumor with the hypoglossal nerve in this position. The vagus nerve (white vessel loop) can be seen coursing directly into the tumor.

Figure 2B: A straight midline incision was used. The mandible was pre-plated and the dissection was then extended posteriorly along the floor of mouth.

Figure 2C: The external carotid artery was displaced anteriorly and is seen running over the tumor, consistent with preoperative imaging.

Figure 2D: Ligation of the external carotid artery permitted delivery of the tumor.

Figure 2E: The final operative site after extirpation is shown with view to the jugular foramen.

Figure 2F: Gross pathology specimen demonstrated a well-encapsulated, round, tan tumor $7.9 \mathrm{~cm}$ in greatest dimension. 


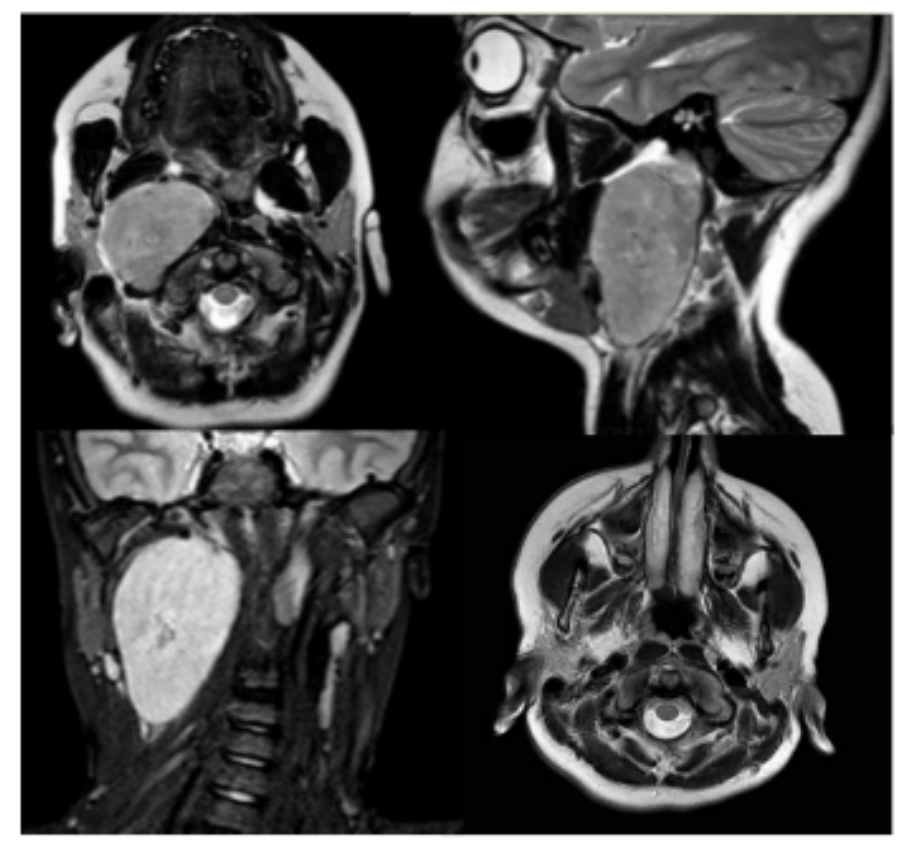




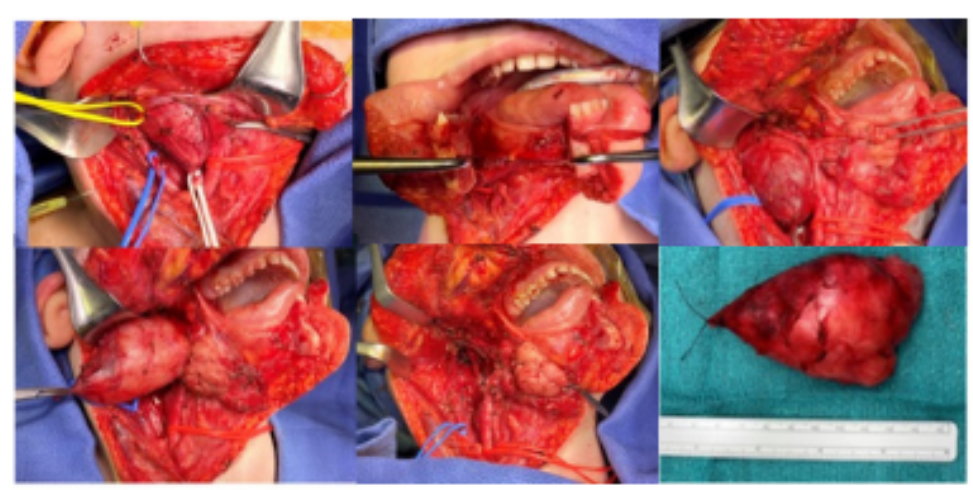

